\title{
Diffraction-Pattern Based on Spontaneous Wrinkled Thin Films
}

\author{
Shi Peng ${ }^{1}$, Wei $\mathrm{Li}^{1,2}$ and Jing Zhang ${ }^{3, *}$ \\ ${ }^{1}$ College of Textile, Donghua University, Shanghai 201620, China \\ ${ }^{2}$ Center for Civil Aviation Composites, Shanghai 201620, China \\ ${ }^{3}$ Department of Applied Physics, Donghua University, Shanghai 201620, China
}

\begin{abstract}
We investigate wrinkled thin films by small angle light scattering and microscope, demonstrating that wrinkled thin films are gratings. The diversity of diffraction patterns is realized by a simple light path through wrinkles. Fourier transform is introduced to explain the position and pattern of diffraction orders. The analysis could be a fast and quantized characterization for complex wrinkles. [doi:10.2320/matertrans.M2016287]
\end{abstract}

(Received August 19, 2016; Accepted October 3, 2016; Published November 28, 2016)

Keywords: $\quad$ wrinkle, diffraction pattern, Fourier transform $(F T)$

\section{Introduction}

Spontaneous wrinkle is usual in nature as fingerprints ${ }^{1)}$, flower petals ${ }^{2}$. Also its sinusoidally buckled surface with a dominant periodicity ${ }^{3,4)}$ keeps spurring interest in engineering. Researches can be roughly divided into two groups concerning to wrinkles' application. First is to be the functional patterned surface, such as superhydrophobic coating ${ }^{5)}$, high light-harvesting efficiency device ${ }^{6}$, template for aligning colloidal particles ${ }^{7)}$ and living cells ${ }^{8)}$, tunable optical grating ${ }^{9)}$ and so on. Second is as a strategy for measuring thickness, elastic moduli, Poisson's ratio, and residual stress ${ }^{10,11)}$ due to its sensitivity to the mechanical and thermal properties of the system.

For wider application of wrinkles, various methods are introduced to guide them as pre-patterning the substrate before coating and mechanical stretching or compression. ${ }^{12,13)}$ Thus abundant and diversified morphologies inspired from basic bulking are displayed. Star-burst pattern are generated by using stripes of UVO lines. ${ }^{4)}$ Concave and convex structures emerge while manipulation the adhesion at the interface. ${ }^{12)}$ Lamellar, hexagonal, herringbone and their variations can also be created by varying the physical properties. ${ }^{14)}$

An effective approach to extract quantitative information from extremely diversified wrinkles patterns is of paramount importance. Small-angle light scattering is a convincing way for automatic pattern recognition, founded on the basic physical phenomenon diffraction. ${ }^{15)}$ In addition, it is a well-established system to get the averaged wavelength of wrinkles since it can minimize measurement error and quickly provide the indispensable information in spite of the diversity and complexity of samples for both off-line and in-situ observation. ${ }^{5,16-19)}$ However, wrinkle surface morphology could also be predicted other than the wavelength by understanding the derivation mechanism from wrinkles to diffraction patterns, which is lacking at present.

In this paper, diffraction pattern and character wavelength and their relevance to several quintessential wrinkles were taken into considerations experimentally and theoretically. Several straightforward models were introduced for getting

*Corresponding author, E-mail: jingzh@dhu.edu.cn the precise understanding. Fast Fourier transform (FFT) and inverse FFT (iFFT) was also introduced. We hope the analysis can provide a guidance to rapidly study both simple and complex wrinkles, and path the way for in situ observing the wrinkle's evolution in small chambers for various preparations.

\section{Experimental Methods}

\subsection{Wrinkles preparation}

In this study, a one-step room-temperature and atmosphere pressure PECVD (Plasma enhanced chemical vapor deposition) was employed to prepare a compressively stressed film with micro scale roughness, without any intentional heat or mechanical stretch. A high voltage power supply of $20 \mathrm{KHz}$ was used for plasma generation between two parallel plate electrodes. Precursor gas of hexamethyldisiloxane (HMDSO, $\left.\mathrm{Si}\left(\mathrm{CH}_{3}\right)_{3}-\mathrm{O}-\mathrm{Si}\left(\mathrm{CH}_{3}\right)_{3}\right)$ maintained at $25^{\circ} \mathrm{C}$ was carried by $\mathrm{Ar}$ by bubbling, together with another two separate lines for $\mathrm{Ar}$ and $\mathrm{O}_{2}$, to a $40 \mathrm{~m} * 40 \mathrm{~mm} * 2 \mathrm{~mm}$ reaction chamber. All flow rates were monitored using mass flow controllers. A $150-\mu \mathrm{m}-$ thick polyester (PET) film with moderately high Young's modulus $(71.7 \mathrm{Gpa})$ was used as the substrate. By controlling gas proportion and power density applied, variously patterned wrinkles were prepared after 2 minutes. However, detailed and exact conditions to obtain a specific pattern were still under research.

\subsection{Diffraction pattern observing}

Schematic illustration for optical system to observe the transmission pattern is showed in Fig. 1. The light source came from a xenon lamp, with output wavelength ranging from 200 to $2000 \mathrm{~nm}$. Firstly it was focused by the convex lens (lens 1) to a pinhole with a radius of $50 \mu \mathrm{m}$, and then again by the camera lens (lens 2). Distances from pinhole to lens 2 and from lens 2 to the plane of sample were set as doubled focal length to make sure the spot projected on the sample keeps the size as pinhole. Transmittance patterns were observed on the white screen. A monochromatic light source with the wavelength of $632.8 \mathrm{~nm}$ was also applied to this system. Inasmuch as red laser light was much easier to focus, pinhole and camera lens were omitted and size of the spot illuminated on samples was assured to keep the same radius as 


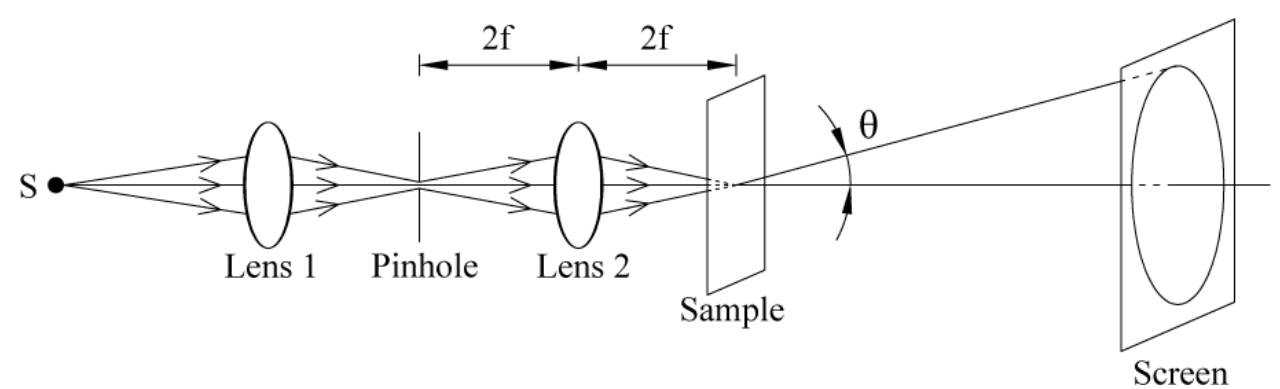

Fig. 1 Optical system for observing Fraunhofer diffraction pattern with white light. Light from a xenon lamp is focused into a pinhole through Lens1, and then illuminates the sample after further focused by a camera lens (lens 2). Diffraction pattern is observed on a white screen. Angle $\theta$ between optical axis and the line drawn from the spot center to a certain point in the screen plane is the diffraction angle.

white light.

\subsection{Surface morphology characterization}

After observing the specific diffraction patterns of wrinkles, the exact locations were marked by slightly scratches from the backside of the substrate to be prepared for the analysis of surface texture. Topography of the deposited films was then observed by an optical microscope. Periodic distances in wrinkles (lattice constant $d$ ) were measured from the microscope images for 50 times and then averaged. FFT from microscope images and iFFT were carried out by Matlab.

\section{Results and Discussion}

Surface morphology was observed by optical microscope and typical patterns of wrinkles are shown in Fig. 2 (a) to (f). Buckling with periodic distance within several micrometers and different orientations, as aligned structure, zigzag and isotropic ones, are chosen to be research objects. Obvious dispersion phenomenon in transmission patterns from white light, as in Fig. 2 (g) to (1), demonstrates the nature of wrinkle is the diffraction grating, the same as some previous works ${ }^{9,20)}$. For oriented lines in one and two directions, as Fig. 2 (a) and (b), two and four dots can be observed on the screen regardless of the midst one, and for zigzags (Fig. 2 (c)), the diffraction pattern is the array of six dots. Moreover, rings with different numbers and diffraction angles (Fig. 2 (j) and (1)), together with arcs (Fig. 2(1)), appeared when light was projected onto random wrinkles. Since diffraction pattern is the two-dimensional (2D) FT of the function of the aperture, FFT directly from microscope images are introduced to verify diffraction analyze in theory. The results, from Fig. 2(l) to (x), pictured here, are in good agreement with the diffraction patterns, which helps us to make sure the microscope images match well with diffraction patterns even though those two observations were carried out separately. Both transmission and FFT patterns reveal strong dependence on original microscope images.

For aligned wrinkles, simplest gratings, the evenly-spacing-dots along the direction perpendicular to wrinkle's orientation (Fig. 2(g) and (m)) are discovered. And the narrower the wrinkle (smaller $d$ ) is, the bigger the diffraction angle and the farther the dots to the center portion will be in diffraction pattern with the same wavelength incident light. Similarly, the wider the wrinkle is, the nearer the dots will be according to eq. (1).

$$
\mathrm{d} \sin \theta=\mathrm{m} \lambda
$$

Average periodic distance in Fig. 2(a) is $2.221 \mu \mathrm{m}$ and hence angle is $16.6^{\circ}$ for the first-order diffraction with $632.8 \mathrm{~nm}$ red light illuminating. From Fig. 2(m), diffraction angle is around $16^{\circ}$, approximately the same as the calculating result. And the corresponding FFT results directly from microscope images in the right side are scaled by $\mathbf{k}_{\mathbf{x}}$ here and it will have maximum while $\mathbf{k}_{\mathbf{x}}=2 \pi / d$. For example, wavelength $d$ in Fig. 2(a) is $2.22 \mu \mathrm{m}$ and thus in FFT the first harmonic appears when $\mathbf{k}_{\mathbf{x}}=0.9 \pi \mathrm{um}^{-1}$, as shown in Fig. 2(s). This also works for all following sample. And when it is assumed that wavelength of incident light is $632.8 \mathrm{~nm}$ as experimental laser, FFT can be deduced easily with rough agreement to diffraction patterns however which is ignored here.

And Fig. 2(b) shows the combination of wrinkles in both horizontal and vertical directions. Diffraction pattern becomes four dots (Fig. 2(h) and (n)). The wider horizontal arranged two dots are from the slightly narrower vertical wrinkles $(1.847 \mu \mathrm{m})$ while vertical laid dots are from horizontal slits $(2.136 \mu \mathrm{m})$. Similarly, from eq. (1), we can get the diffraction angle $\theta$ as $20.0^{\circ}$ and $17.2^{\circ}$ individually. And white dots in FFT from microscope images are located at $\pi \mathrm{um}^{-1}$ and $0.9 \pi \mathrm{um}^{-1}$.

From the lesson of Fig. 2(b), we come to realize that diffraction pattern of multi slits with different orientations should be the superposition of the dots from all directions. Here for zigzag pattern shown in Fig. 2(c), we can simply divide them into two individual groups of short wrinkles (lines) with different orientations. Thus it becomes easier to envision the diffraction pattern, which should be combination of the dots along the directions perpendicular to both parts of the zigzag's orientation. However, from the experimental results, another two dots with strong intensity appeared, as the blue circled dots in Fig. 2(i) and (o). Therefore, we calculate the two-dimensional Fourier transform of the zigzag function by a simple model to analyze its diffraction pattern in detail.

Simple zigzag model is as Fig. 3(a) and we let the aperture consist of a grating, that is, number $\mathrm{N}$ along the $\mathrm{x}$ direction and $\mathrm{M}$ along $\mathrm{y}$ of the parallel units of length $d_{1}$ and of width $d_{2}$. Height of the zigzag $a$ can be changed from 0 to $d_{1}$ in the $\mathrm{x}$-axis while the inclining angle of each part in the zigzag keeps equal, $\beta_{1}=\beta_{2}$, by fixing $d_{2} / 2$ in the $y$-axis, according to the optical microscope image in Fig. 2 (c). The function of the grating can be defined as $\mathrm{y}_{1}=d_{2}-d_{2} \mathrm{x} /(2 a)$ and $\mathrm{y}_{2}=d_{2} \mathrm{x} /$ (2a) individually. 

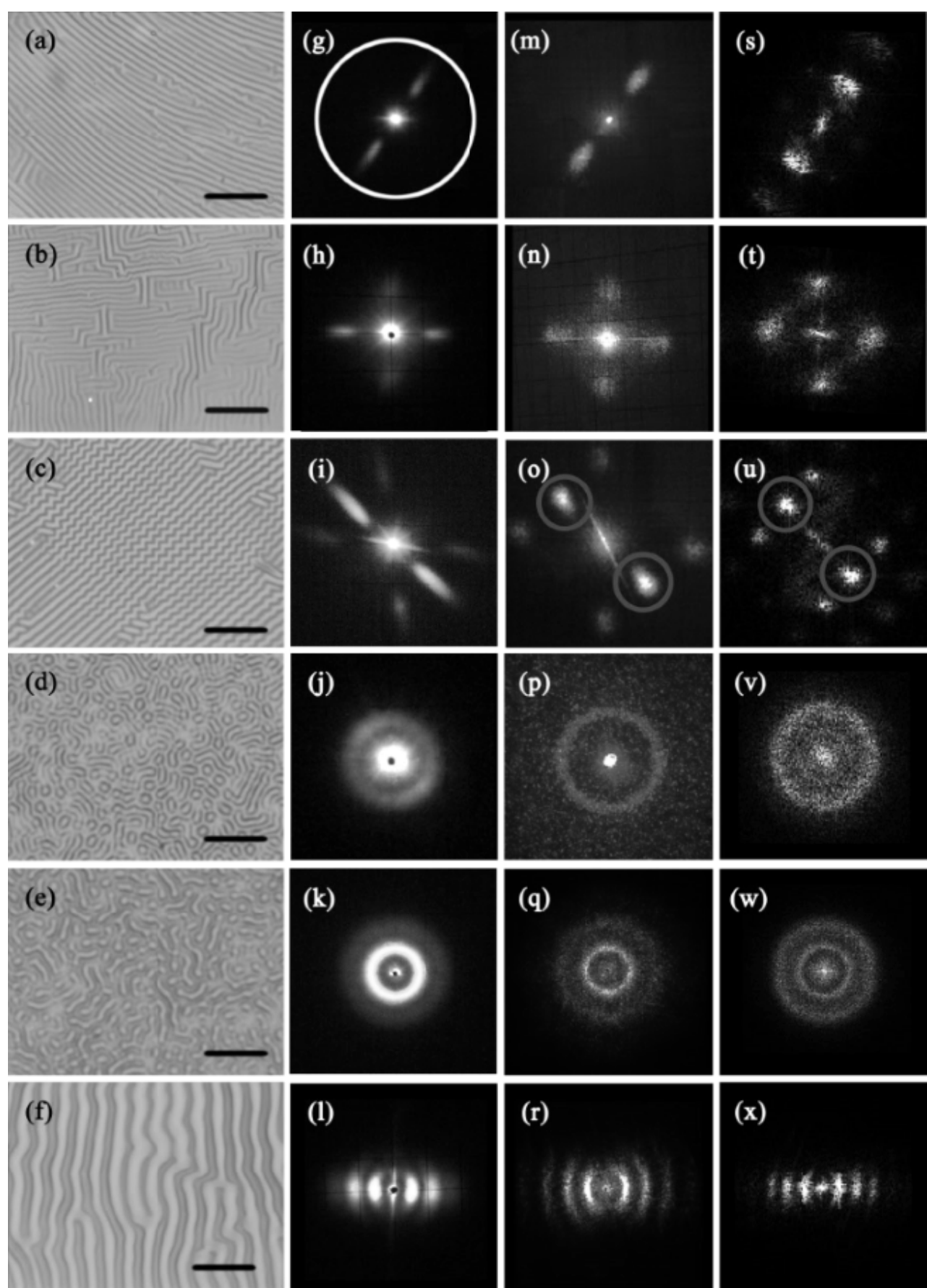
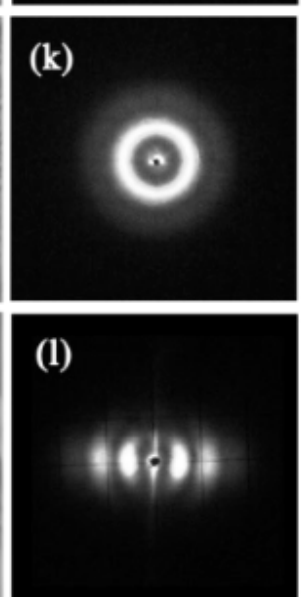
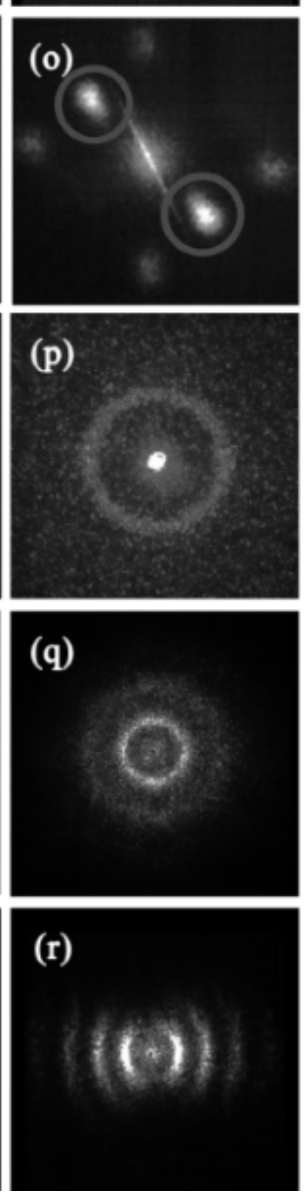
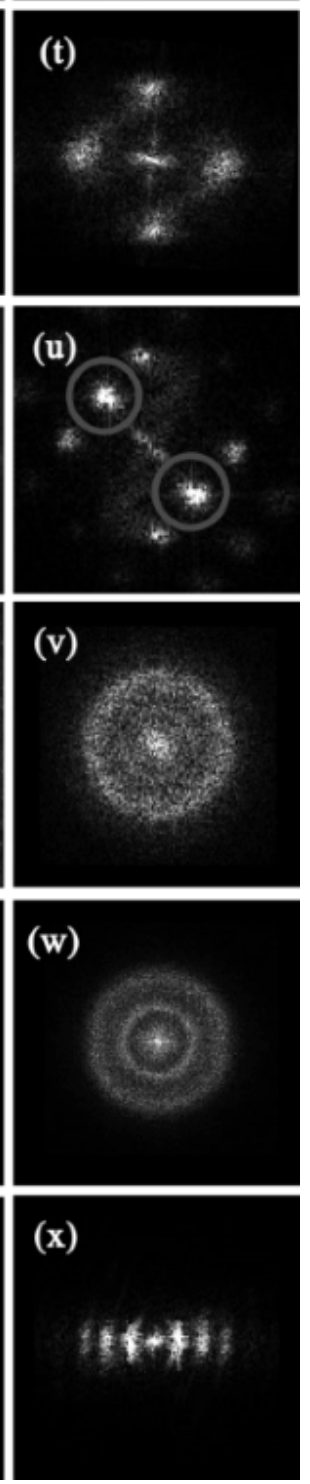

Fig. 2 Optical Microscope images for representative patterns and corresponding diffraction patterns from white and red light source, the second and third line. For (a) (b) (f) the scale bar is $20 \mu \mathrm{m}$ and (c) (e) (d) is $10 \mu \mathrm{m}$. White circle in (g) is the diffraction angle of $25^{\circ}$ and it works for all the diffraction patterns. Corresponding Fast Fourier Transform by Mathematica are listed in the fourth line with the scale bar of $1 \mu \mathrm{m}^{-1}$.

By integration, the results of the diffraction intensity, as following eqs. (2) to (4), are the production of interference factor $\mathrm{I}_{\mathrm{Int} .}$ and diffraction factor $\mathrm{I}_{\text {Dif., }}$, as well known.

$$
\mathrm{I}=\mathrm{I}_{\text {Int. }} . \mathrm{I}_{\text {Dif. }} \text {. }
$$

$$
\mathrm{I}_{\text {Int. }} \propto\left[\frac{\sin \left(\mathrm{k}_{\mathrm{x}} \mathrm{d}_{1} \mathrm{~N} / 2\right)}{\sin \left(\mathrm{k}_{\mathrm{x}} \mathrm{d}_{1} / 2\right)} \frac{\sin \left(\mathrm{k}_{\mathrm{y}} \mathrm{d}_{2} \mathrm{M} / 2\right)}{\sin \left(\mathrm{k}_{\mathrm{y}} \mathrm{d}_{2} / 2\right)}\right]^{2}
$$

$$
\begin{aligned}
\mathrm{I}_{\text {Dif. }} \propto\left[\frac{\sin \frac{\left(\mathrm{k}_{\mathrm{x}} a+\mathrm{k}_{\mathrm{y}} \frac{\mathrm{d}_{2}}{2}\right)}{2}}{\frac{\mathrm{k}_{\mathrm{x}}+\mathrm{k}_{\mathrm{y}} \frac{\mathrm{d}_{2}}{2 a}}{2}}+\cos \left(\mathrm{k}_{\mathrm{y}} \mathrm{d}_{2} / 2\right) \frac{\sin \frac{\mathrm{k}_{\mathrm{x}} a-\mathrm{k}_{\mathrm{y}} \frac{\mathrm{d}_{2}}{2}}{2}}{\frac{\mathrm{k}_{\mathrm{x}}-\mathrm{k}_{\mathrm{y}} \frac{\mathrm{d}_{2}}{2 a}}{2}}\right]^{2} \\
+\left[\sin \left(\mathrm{k}_{\mathrm{y}} \mathrm{d}_{2} / 2\right)\right]^{2}\left[\frac{\sin \frac{\mathrm{k}_{\mathrm{x}} a-\mathrm{k}_{\mathrm{y}} \frac{\mathrm{d}_{2}}{2}}{2}}{\frac{\mathrm{k}_{\mathrm{x}}-\mathrm{k}_{\mathrm{y}} \frac{\mathrm{d}_{2}}{2 a}}{2}}\right]^{2}
\end{aligned}
$$

Typical pattern for interference intensity $\mathrm{I}_{\text {Int. }}$ is the array of dots showed as white dots on the left of Fig. 3(c) with some other weaker dots between the strongest ones appearing and the numbers of them being N-2 and M-2. However, these dots 
(a)

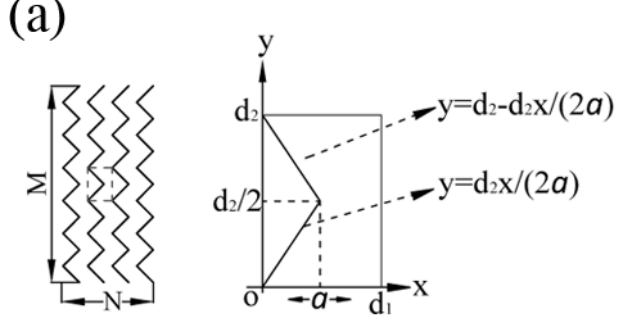

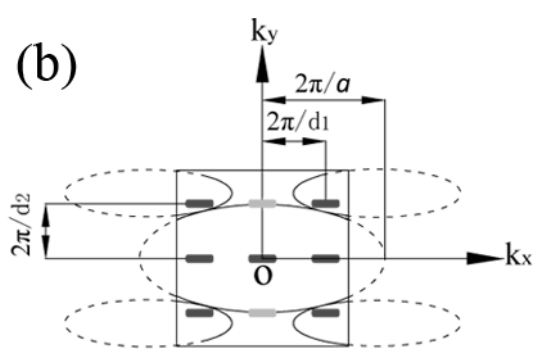

(c)
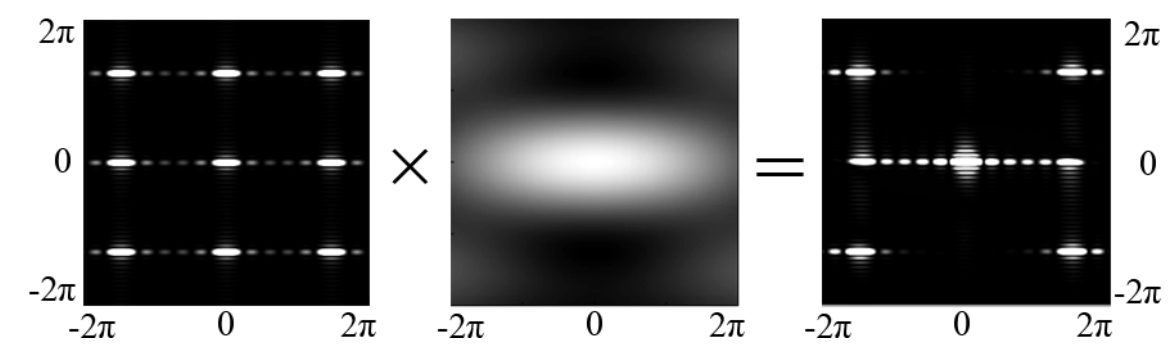

Fig. 3 Simple Model for Zigzag and the results from the calculation. For $(\mathrm{c})$, each parameter is from the real sample Fig. $2(\mathrm{c})$, $\mathrm{d}_{1}=1.361, \mathrm{~d}_{2}=1.175 \mathrm{~d}_{1}, a=$ $0.387 d_{1}, N=6, M=20$.

are not observed in experiment because of the weaker intensity. Pattern for diffraction part $\mathrm{I}_{\text {Dif. }}$ is similar to ellipse as the middle one in Fig. 3 (c) and its product with $\mathrm{I}_{\text {Int. }}$ turns to be finally result. Detailed and clear results are revealed in Fig. 3 (b), the rough contour from calculations, and from eq. (3) we can get the conclusion that distances between strongest interference dots are affected by lattice constant $d$, here determined by the $2 \pi / d_{1}$ and $2 \pi / d_{2}$ for $\mathrm{x}$-axis and y-axis individually. And for diffraction apart, when we put $\mathbf{k}_{\mathbf{x}}=0, \mathbf{k}_{\mathbf{y}}=0$ separately and we can get the solution for $\mathrm{I}_{\text {Dif. }}=0, \mathbf{k}_{\mathbf{x}}=2 \pi / a$ and $\mathbf{k}_{\mathbf{y}}=2 \pi / \mathrm{d}_{2}$ accordingly. In this case, $a \leq d_{1}<d_{2}$, it means $2 \pi / d_{1} \leq 2 \pi / a$. So that's why the two dots in blue circles in Fig. 2 (i) \& (o) appear here. And based on eq. (1), those two dots should lie at the diffraction angle of approximately $27.7^{\circ}$. Other four shinning dots on the diagonal are at the angle of $36.3^{\circ}$.

Since the Fourier transform preserves rotation, if the input pattern is rotated, the diffraction pattern is rotated as the same the angle but the remaining otherwise keeps unchanged. Then just rotating the simple zigzag model (Fig. 3(a)) and the result (Fig. 3(c)) $30^{\circ}$ in the clockwise direction, it fits well with the experimental patterns, as showed in Fig. 2(i) and 2(o).

In above samples, oriented lines, explicitly or implicitly, are taken into discussion. However, in this section we will analyze the most common random wrinkles without any particular orientations, as shown in Fig. 2 (d) and (e), for which transmission patterns tend to be one-ring or two-ring. It might be difficult to envision its diffraction pattern since we are easy to be disturbed by such irregular and isotropic domains. But fine and subtle features can be extracted by further observation and explanation. Figure 4 is the contour line from isotropic wrinkles and several red arrows are drawn here since by elaborative observations several oriented buckling can be discovered in local range. In this sense diffraction pattern should be the dots lie in the direction perpendicular to the grating, as the red arrows, which would appear in several different orien-

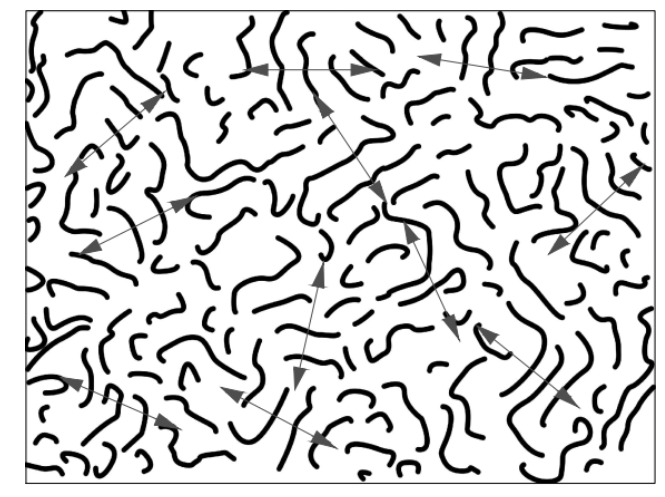

Fig. 4 Explanation for diffraction pattern from irregular orientation wrinkle. Black lines are the actual contour from part of Fig. 2(d). Red arrows show the possible directions for diffraction.

tations. Hence from all wrinkles, diffraction dots could appear from $0^{\circ}$ to $360^{\circ}$. In addition spacing in frequency domain is always inversely proportional to the distance between adjacent crests. So if the periodic distance remains consistent, the diffraction dots would appear in all the directions with the same spacing. Then rings will appear which can be proved by Fig. 2(d) and its diffraction pattern as Fig. 2(j), (p) and (v).

Quantitative analysis for random and isotropic wrinkles can draw lessons from above samples by decomposing them into local-scoped aligned lines as aforementioned. For simplifying the calculation, another hypothesis should be stated here. Distance between adjacent wrinkles from all directions is homogenous and always keeps a value. Then from eq. (1), location of the peak value of diffraction intensity could be easily derived. However, obvious difference appears in the results of both calculations and experiments for two similar microscope images, as shown in Fig. 2 (d), (e), (p) and (q). Meanwhile, $d$ (distance between adjacent wrinkles) and the location of peak value $k_{x}$ in FFT results did not match the 


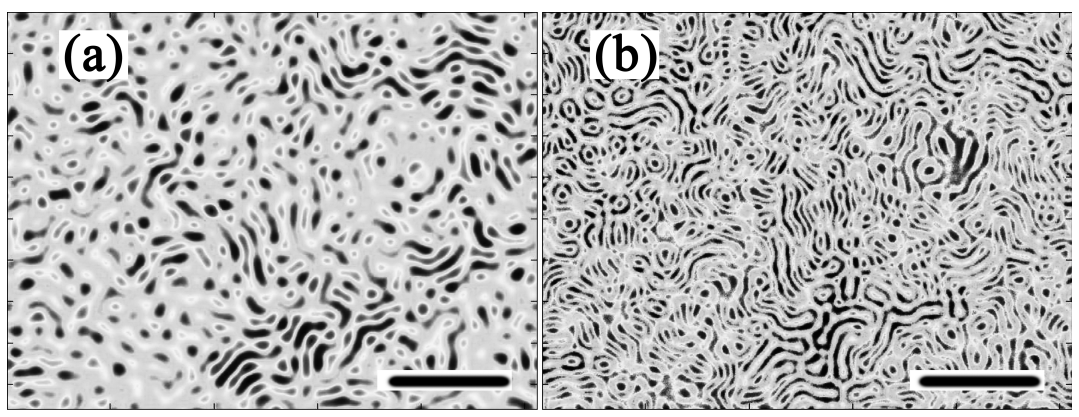

(c) Wrinkle

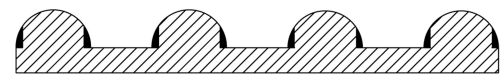

Diffraction constant in wrinkle

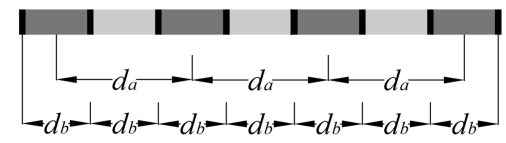

Fig. 5 Inverse FFT of different order of rings in Fig. 2 (w). (a) iFFT from the inner ring, (b) iFFT from the outer ring. Scale bar is $10 \mu$ m. (c) Lattice constant $d$ in upon images.

general rule $k_{x}=2 \pi / d$ during the calculations.

For getting an idea of the reason of two-ring and one-ring, part of the FFT spectrum was zeroed and then iFFT was carried out for the each ring in two-ring case. Figure 5 exhibits the detailed results while (a) and (b) are the iFFT results from the inner and outer halo in Fig. 5 (w) individually, from which we get the conclusion that the wrinkle might be composed of three parts with different transmittance, other than simply transparent and opaque ones, shown in Fig. 5 (c). In this case, two lattice constants can be obtained and then two rings appear. The inner ring in Fig. 2(w) located at $\pi \mu \mathrm{m}^{-1}$ should be the superstition of points from isotropic grain with a larger wavelength $(1.865 \mu \mathrm{m})$ from $0-360^{\circ}$ directions. And the outer ring at $2.3 \pi \mu \mathrm{m}^{-1}$ should be the combination of dots from edges of the buckling $(0.863 \mu \mathrm{m})$. Thus two-ring appears here because FFT is totally liner and the filtered image could be the superposition of all subsections. Same reasoning will go for Fig. 2 (d). However, only a bigger halo from edges of wrinkles can be observed here since it is not easy to extract the obvious difference of the wrinkle and flat parts.

Finally, last example in Fig. 2 would be explained quickly. Pattern in Fig. 2 (f) depicts a diffraction grating with a much wider $d$ than any other examples and hence more oscillations could be observed, according to eq. (1). Here $d$ owns a value of $5.293 \mu \mathrm{m}$ and first diffraction angle locates at $6^{\circ}$ when a red light was projected, and the second and third order angle at about $12^{\circ}$ and $18^{\circ}$ individually, as Fig. 2 (r). Another distinct feature of this sample should be the arcs in both diffraction pattern and calculated results which attributes to the winding grating instead of totally straight ones as in Fig. 2 (a), or isotropic ones in Fig. 2(d) and (e).

\section{Conclusions}

In this study various diffraction pattern are observed and detailed transform mechanism is discussed. For simply oriented wrinkles and isotropic ones, $d=2 \pi / \mathrm{k}$ or $\boldsymbol{d}=\mathbf{m} \lambda / \sin \boldsymbol{\theta}$ can work well. But the periodic distance $d$ is of paramount importance here and it is easy to make a faulty judgment with insufficient analysis. Meanwhile, how to expect wrinkle pattern from diffraction pattern is fully explained here, which may be helpful to in situ observe wrinkles formation and evolution.

\section{Acknowledgments}

This work is supported by the Innovation Funding Program for Ph.D in Donghua University (CUSF-DH-D-2014010).

\section{REFERENCES}

1) M. Kucken: Forensic Sci. Int. 171 (2007) 85-96.

2) R.L.A. Kourounioti, et al:: J. R. Soc. Interface 10 (2013).

3) D.B.H. Chua, H.T. Ng and S.F.Y. Li: Appl. Phys. Lett. 76 (2000) 721723.

4) E.P. Chan and A.J. Crosby: Soft Matter 2 (2006) 324-328.

5) Y. Rahmawan, et al.: Langmuir 26 (2010) 484-491.

6) J.B. Kim, et al.: Nat. Photonics 6 (2012) 327-332.

7) C.H. Lu, H. Mohwald and A. Fery: Soft Matter 3 (2007) 1530-1536.

8) P. Uttayarat, et al.: J. Biomed. Mater. Res. A 75A (2005) 668-680.

9) C.J. Yu, et al.: Appl. Phys. Lett. 96 (2010).

10) C.M. Stafford, et al.: Nat. Mater. 3 (2004) 545-550.

11) J.Y. Chung, A.J. Nolte and C.M. Stafford: Adv. Mater. 23 (2011) 349368.

12) P.J. Yoo and H.H. Lee: Langmuir 24 (2008) 6897-6902.

13) Yague, J.L., et al.: Design of Ordered Wrinkled Patterns with Dynamically Tuned Properties, Nineteenth European Conference on Chemical Vapor Deposition (Eurocvd 19), (2013). 46: pp. 40-45.

14) M. Guvendiren, S. Yang and J.A. Burdick: Adv. Funct. Mater. 19 (2009) 3038-3045.

15) G.G. Lendaris and G.L. Stanley: Proceedings of the Institute of Electrical and Electronics Engineers 58 (1970) 198.

16) M.D. Casper, et al:: Soft Matter 9 (2013) 7797-7803.

17) C.M. Chen, J.C. Reed and S. Yang: Soft Matter 9 (2013) 11007-11013.

18) S.J. Kwon, J.H. Park and J.G. Park: Phys. Rev. E Stat. Nonlin. Soft Matter Phys. 71 (2005).

19) A.J. Nolte, R.E. Cohen and M.F. Rubner: Macromolecules 39 (2006) 4841-4847.

20) C. Harrison, et al.: Appl. Phys. Lett. 85 (2004) 4016-4018. 